\title{
Central Limit Theorem in Multitype Branching Random Walk
}

\author{
A. Rahimzadeh Sani \\ Tarbiat Moallem University
}

\begin{abstract}
A discrete time multitype ( $p$-type) branching random walk on the real line $\mathcal{R}$ is considered. The positions of the $j$-type individuals in the $n$-th generation form a point process. The asymptotic behavior of these point processes, when the generation size tends to infinity, is studied. The central limit theorem is proved.
\end{abstract}

Keywords. Central limit theorem; counting measure; intensity measure; multitype branching random walk.

\section{Introduction}

A discrete time multitype ( $p$-type) branching random walk on the real line $\mathcal{R}$ is considered. The process starts with a single $i$-type ancestor located at the origin. This particle splits into a random number of new particles of different types and located at random places, with probability law depending on $i$, to make the first generation individuals. Each of these particles splits in the same way and the process goes on as there are individuals alive. For each fixed $i=1, \ldots, p$, let $Z_{i}^{n}(\cdot)=\left(Z_{i 1}^{n}(\cdot), \ldots, Z_{i p}^{n}(\cdot)\right)$ be the vector of counting measures related to the point processes that give the positions of different types of individuals in generation $n$ descended from an $i$-type one in generation zero. The purpose of this study is to establish a central limit theorem for the counting measures $\left\{Z_{i j}^{n}(\cdot)\right\}$. There are a number of works proving a central limit theorem for the counting measures in branching random walk; see Biggins (1992), Biggins and Rahimzadeh (2005), Bramson et al. (1992) and Rahimzadeh (2008).

The key point in this paper is showing that, the counting measure of the $n$-th generation individuals $Z_{i j}^{n}(\cdot)$ behave the same as its intensity measure, 
as $n \rightarrow \infty$. Then we use the central limit theorem for the intensity measure of $Z_{i j}^{n}(\cdot)$, from Rahimzadeh (2008), to get our results here. This is a well known technique in literature, for example Bramson et al. (1992), and we will use it here. The central and local limit theorems for intensity measures (that we need here) are proved in Rahimzadeh (2008). We use the notations and the results of that paper. In fact, the technique of the present work made it necessary to create Rahimzadeh (2008). There is a similar result for counting measures in Biggins and Rahimzadeh (2005), which is based on the convolution of measures. But we give a new proof with a different technique here. This paper consists of three sections. Section 1 is introduction. In Section 2 we give the notations and the definition of tilted versions of $Z_{i j}^{n}(\cdot)$ with the main results. Section 3 contains the proof of the main results.

\section{Notations and Some Preliminary Results}

Similar to Biggins and Rahimzadeh (2005) we consider a multitype branching random walk on the real line $\mathcal{R}$. The process starts with a single $i$-type ancestor located at the origin. Let $\left\{Z_{i}^{n}(\cdot)\right\}=\left\{\left(Z_{i 1}^{n}(\cdot), \ldots, Z_{i p}^{n}(\cdot)\right)\right\}$ be the vector of counting measures related to the types in generation $n$. For each fixed $i, j, Z_{i j}^{n}(\cdot)$ is the counting measure of $j$-type individuals in generation $n$ related to the point process $\left\{Z_{i j}^{n}(s): s=1,2, \ldots\right\}$, where $Z_{i j}^{n}(s)$ is the position of $s$-th person of $j$-type individuals in generation $n$. So for any Borel measurable set $A \subset \mathcal{R}$,

$$
Z_{i j}^{n}(A)=\int_{A} Z_{i j}^{n}(d x)=\sum_{Z_{i j}^{n}(s) \in A} 1,
$$

which is almost surely finite. Let $\mu_{i j}^{1 *}(\cdot)$ be the intensity measure related to the counting measure $Z_{i j}^{1}(\cdot)$, so for any Borel measurable set $A \subset \mathcal{R}, \mu_{i j}^{1 *}(A)=$ $E\left[Z_{i j}^{1}(A)\right]$. The point process $Z_{i j}^{n}$ has the intensity measure $\mu_{i j}^{n *}$, defined inductively, if $\mu^{n *}=\left\{\mu_{i j}^{n *}\right\}_{p \times p}$ then $\mu_{i j}^{(n+1) *}=\sum_{k=1}^{p} \mu_{i k}^{1 *} \otimes \mu_{k j}^{n *}$ where $\otimes$ is the ordinary convolution of measures. By induction for any measurable set $A, \mu_{i j}^{n *}(A)=E\left[Z_{i j}^{n}(A)\right]$. Define the Laplace transforms $m_{i j}(\lambda)$ with complex arguments $\lambda$ by:

$$
m_{i j}(\lambda)=\int_{\mathcal{R}} e^{-\lambda x} \mu_{i j}(d x), \quad \lambda \in \mathcal{C} .
$$


Let $L=\bigcap_{i, j} \operatorname{int}\left\{\lambda=\theta+\boldsymbol{i} \eta \in \mathcal{C}: m_{i j}(\theta)<\infty\right\}$. Then $L$ is an open convex subset of $\mathcal{C}$ and $L_{0}=L \cap \mathcal{R}$ is an open interval (see Biggins and Rahimzadeh, 2005). For each $\lambda \in L$, define $M(\lambda)=\left\{m_{i j}(\lambda)\right\}_{p \times p}$ and let $M^{n}(\lambda)=\left\{m_{i j}^{n}(\lambda)\right\}_{p \times p}$ be its $n$-th power. Then we have

$$
m_{i j}^{n}(\lambda)=\int_{\mathcal{R}} e^{-\lambda x} \mu_{i j}^{n *}(d x)=E\left[\sum_{s} e^{-\lambda Z_{i j}^{n}(s)}\right] .
$$

The entries of the matrix $M(\lambda)$ are complex-valued analytic functions in $\lambda$; and for those values of $\lambda=\theta \in L_{0}$, the matrix $M(\lambda)$ has all non-negative entries. The matrix $A$ is called positive regular if all the entries are nonnegative real numbers and for some $n$, all the entries of $A^{n}$ are positive (see Mode, 1971, Ch. 1.6). Assume:

$\mathcal{A}(1)$ : The process $\left\{Z_{i}^{n}\right\}$ is positive regular in the sense that the matrix $M=\left\{m_{i j}(\theta)\right\}$ for $\theta \in L_{0}$ is positive regular.

So, the conditions of Theorem 1 in Biggins and Rahimzadeh (2005) hold and this Theorem implies that, there is an open set $\Omega \subset L \cap \mathcal{R}$ such that, for any $\theta \in \Omega, M(\theta)$ is positive regular and has a simple maximum eigenvalue $\rho(\theta)$, with related left and right eigenvectors $u(\theta)$ and $v(\theta)$ which are analytic in $\theta \in \Omega$ (infinitely differentiable). They are strictly positive and normalized so that $u(\theta)^{T} v(\theta)=\sum_{i=1}^{p} u_{i}(\theta) v_{i}(\theta)=1$, and $\sum_{i=1}^{p} u_{i}(\theta)=1$. Also for any $\theta \in \Omega, \lim _{n \rightarrow \infty} \rho(\theta)^{-n} m_{i j}^{n}(\theta)=v_{i}(\theta) u_{j}(\theta)$.

The multitype branching random walk is strongly non-lattice when it is positive regular and, for some $(k, l)$ and some $\theta \in \Omega$,

$$
\left|\frac{m_{k l}(\theta+\mathbf{i} \eta)}{m_{k l}(\theta)}\right|=1 \quad \text { only when } \quad \eta=0 .
$$

When the process is strongly nonlattice $\rho(\theta)$ is strictly log-convex in $\theta \in \Omega$ (see Biggins and Rahimzadeh, 2005). We set the next assumption:

$\mathcal{A}(2)$ : The process $\left\{Z_{i}^{n}\right\}$ is strictly non-lattice.

For any $\theta \in \Omega$, define $\Lambda(\theta)=\log \rho(\theta)$ then by $\mathcal{A}(2), \sigma^{2}=\Lambda^{\prime \prime}(\theta)>0$. For $\alpha \in(1,2]$ define $\kappa=\kappa(\theta)=\rho(\alpha \theta) /|\rho(\theta)|^{\alpha}$. Then $\kappa$ is a strictly positive continuous function in $\theta \in \Omega_{\alpha}=\{\theta \in \Omega: \alpha \theta \in \Omega\}$. Assume

$\mathcal{A}(3): \quad$ For $\alpha \in(1,2]$ and $\theta \in \Omega, \kappa=\kappa(\theta)=\frac{\rho(\alpha \theta)}{|\rho(\theta)|^{\alpha}}<1$.

When $\mathcal{A}(3)$ holds, the process is supercritical in the sense that, $\rho(\theta)>1$, 
(see Biggins and Rahimzadeh, 2005). For each $\theta \in \Omega$, $i$, and $n$, define

$$
W_{i}^{n}(\theta)=\sum_{j=1}^{p} \int_{\mathcal{R}} \frac{v_{j}(\theta) e^{-\theta x}}{v_{i}(\theta) \rho(\theta)^{n}} Z_{i j}^{n}(d x) .
$$

Then $\left\{W_{i}^{n}(\theta)\right\}$ is a non-negative martingale with respect to $\left\{\mathcal{F}_{n}\right\}$, where each $\mathcal{F}_{n}$ is the $\sigma$-algebra containing all information of all generations up to generation $n$. So it converges almost surely to $W_{i}(\theta)$ and for all $n, E\left[W_{i}^{n}(\theta)\right]=1$ (see Theorem 2 in Biggins and Rahimzadeh, 2005). We assume:

$\mathcal{A}(4)$ : For some $\theta \in \Omega$ and $\alpha \in(1,2]$,

$$
\beta(\theta)=\max \left\{E\left[W_{i}^{1}(\theta)^{\alpha}\right] \quad: \quad i=1, \ldots, p\right\}<\infty .
$$

$\mathcal{A}(5): 0 \in \Omega$.

When $\mathcal{A}(4)$ holds for some $\theta_{0} \in \Omega, \beta$ is a finite real valued continuous function in a neighborhood of $\theta_{0}$ in $\Omega$. Same as in Rahimzadeh (2008), for any fixed $\theta \in \Omega$, we define the tilted measures $Z_{\theta, i j}^{n}(\cdot)$ and $\mu_{\theta, i j}^{n *}(\cdot)$ by

$$
Z_{\theta, i j}^{n}(d x)=\frac{v_{j}(\theta)}{v_{i}(\theta)} \cdot \frac{e^{-\theta x}}{\rho(\theta)^{n}} Z_{i j}^{n}(d x)
$$

and

$$
\mu_{\theta, i j}^{n *}(d x)=\frac{v_{j}(\theta)}{v_{i}(\theta)} \cdot \frac{e^{-\theta x}}{\rho(\theta)^{n}} \mu_{i j}^{n *}(d x)
$$

The mean drift of the measure $\mu_{\theta}=\left\{\mu_{\theta, i j}^{1 *}\right\}$ is $a=-\rho^{\prime}(\theta) / \rho(\theta)$, (see Bramson et al., 1992). Define the centered shifted measures $\bar{Z}_{\theta, i j}^{n}(d x)=$ $Z_{\theta, i j}^{n}(n a+d x)$ and $\bar{\mu}_{\theta, i j}^{n *}(d x)=\mu_{\theta, i j}^{n *}(n a+d x)$ with $\bar{\mu}_{\theta, i j}(d x)=\mu_{\theta, i j}(a+d x)$. The measures $\mu_{\theta, i j}^{n *}(d x)$ and $\bar{\mu}_{\theta, i j}^{n *}(d x)$ can be interpreted as the intensity measures of $Z_{\theta, i j}^{n}(d x)$ and $\bar{Z}_{\theta, i j}^{n}(d x)$, respectively in the sense that, for any measurable set $A \subset \mathcal{R}$,

$$
\mu_{\theta, i j}^{n *}(A)=E\left[Z_{\theta, i j}^{n}(A)\right] \quad \text { and } \quad \bar{\mu}_{\theta, i j}^{n *}(A)=E\left[\bar{Z}_{\theta, i j}^{n}(A)\right] .
$$

For $\theta \in \Omega$, the matrix $P=\left\{p_{i j}\right\}_{p \times p}=\left\{\bar{\mu}_{\theta, i j}(\mathcal{R})\right\}=\left\{\mu_{\theta, i j}(\mathcal{R})\right\}$ is a positive regular stochastic matrix with stationary measure (see Biggins and Rahimzadeh, 2005):

$$
\pi(\theta)=\left(\pi_{1}(\theta), \ldots, \pi_{p}(\theta)\right), \quad \pi_{k}(\theta)=u_{k}(\theta) v_{k}(\theta), \quad(k=1, \ldots, p) .
$$

Since $P$ is positive regular, so for large values of $n, P^{n}=\left\{p_{i j}^{n}\right\}$ has all 
positive entries. We assume:

$\mathcal{A}(6):$ For each $i, j$ and $\theta \in \Omega$, the characteristic function of the measure, $\bar{\mu}_{\theta, i j}^{n *}(\cdot) / p_{i j}^{n}$ is absolutely integrable.

When $\mathcal{A}(6)$ hold the density function of $\bar{\mu}_{\theta, i j}^{n *}(\cdot) / p_{i j}^{n}$, denoted by $g_{i j}^{n}$, exists. Now we set our main results, Theorems 1 and 2 :

Theorem 11 Let for a fixed $\theta \in \Omega$ and $\alpha \in(1,2], \mathcal{A}(1)-\mathcal{A}(6)$ hold, and $\sigma^{2}=(\log \rho(\theta))^{\prime \prime}>0$. Then for any bounded measurable set $A \subset \mathcal{R}$, and fixed $i, j$,

$$
\lim _{n \rightarrow \infty} \sqrt{2 \pi n} \sigma Z_{\theta, i j}^{n}(n a+A)=u_{j}(\theta) v_{j}(\theta) W_{i}(\theta)|A|
$$

almost surely where $|A|$ is the Lebesgue measure of $A$ and $a=-\rho^{\prime}(\theta) / \rho(\theta)$.

In the next theorem we apply the Riemman integral estimate in the Theorem 1 to get a central limit theorem for the measures $\left\{Z_{i j}^{n}(\cdot)\right\}$. The proof of this theorem is similar to Theorem 2.4 in Rahimzadeh (2008) where the same techniqe is used to prove the central limit theorem for intensity measures $\mu_{i j}^{n *}(\cdot)$.

Theorem 12 Suppose $\mathcal{A}(1)-\mathcal{A}(6)$ hold, $\theta \in \Omega$ and $\sigma^{2}=(\log \rho(\theta))^{\prime \prime}>0$. Then for any bounded measurable set $A \subset[-b, b]$ and all fixed $i, j$,

$$
\lim _{n \rightarrow \infty}\left[\sqrt{2 \pi n} e^{-n \Lambda^{*}(a)} \sigma Z_{i j}^{n}(n a+A)\right]=u_{j}(\theta) v_{j}(\theta) W_{i}(\theta) \int_{A} e^{\theta x} d x,
$$

almost surely where $a=-\rho^{\prime}(\theta) / \rho(\theta)$ and $\Lambda^{*}(a)=-\rho^{\prime}(\theta) / \rho(\theta)+\log \rho(\theta)$.

We conclude this section by two more lemmas that will be needed later. The next Lemma is a local limit theorem result for the tilted intensity measures $\bar{\mu}_{\theta, i j}^{n *}$, which is Theorem 2.2 in Rahimzadeh (2008):

Lemma 1 Suppose $\theta \in \Omega, \alpha \in(1,2]$ be fixed, $a=-\rho^{\prime}(\theta) / \rho(\theta), \sigma^{2}=$ $(\log \rho(\theta))^{\prime \prime}$ and $\mathcal{A}(1)-\mathcal{A}(6)$ hold. Let also $g_{i j}^{n}$ and $f_{\sigma}$ be the density function of $\bar{\mu}_{\theta, i j}^{n *}(\cdot) / p_{i j}^{n}$ and normal distribution $\mathrm{N}\left(0, \sigma^{2}\right)$, respectively. Then for all $i, j$,

$$
\lim _{n \rightarrow \infty}\left|\sqrt{n} g_{i j}^{n}(x \sqrt{n})-f_{\sigma}(x)\right|=0
$$

uniformly in $x \in \mathcal{R}$.

The next lemma is Lemma 3 (iv) in Biggins and Rahimzadeh (2005).

Lemma 2 Suppose for $\alpha \in(1,2]$ and $\theta_{0} \in \Omega, \mathcal{A}(1)-\mathcal{A}(4)$ hold. Then there is a neighborhood of $\theta_{0}$, say $B=B\left(\theta_{0}, \delta\right) \subset \Omega$, such that

$$
\sup \left\{E\left|W_{i}^{n}(\theta)\right|^{\alpha}: \theta \in B, i=1, \ldots, p, n=0,1,2, \ldots\right\}<\infty .
$$




\section{Proofs of the Main Results}

In this section we use $v_{i}, u_{i}$ and $\rho$ for $v_{i}(\theta), u_{i}(\theta)$ and $\rho(\theta)$ for shortening the text. Also for some $\beta>3$ we assume $l=\left[n^{1 / \beta}\right]$ then for large values of $n, 1 \leqslant l<n$ and as $n \rightarrow \infty$, we get $l, n-l \rightarrow \infty$. In this section we assume $n$ to be large enough to have $1 \leqslant l<n$ and for all $i, j, p_{i j}^{n}>0$. By looking at the individuals of the $n$-th generation as the $(n-l)$-th generation children of the people in generation $l$, we use $Z_{k j}^{n}(\cdot \mid l, s)$ as the counting measure related to the point process giving the positions of $j$-type individuals in generation $n$ descended from the $s$-th person with type $k$ in generation $l$. By the branching property of the process, given $\mathcal{F}_{l}$, for all $i, j, k, l$, and $s$ the counting measure $\left\{Z_{k j}^{n}\left(t-Z_{i k}^{l}(s) \mid l, s\right): t=1,2, \ldots\right\}$ is an independent and identical copy of $\left\{Z_{k j}^{n-l}(t): t=1,2, \ldots\right\}$. So for any measurable set $A$, we can write

$$
\begin{aligned}
Z_{\theta, i j}^{n}(A) & =\int_{A}\left[\frac{v_{j} e^{-\theta x}}{v_{i} \rho^{n}}\right] Z_{i j}^{n}(d x) \\
& =\sum_{k=1}^{p} \int_{\mathcal{R}} \frac{v_{k} e^{-\theta y}}{v_{i} \rho^{l}}\left[\int_{A}\left[\frac{v_{j} e^{-\theta(x-y)}}{v_{k} \rho^{n-l}}\right] Z_{k j}^{n}(d x-y \mid l, y)\right] Z_{i k}^{l}(d y) \\
& =\sum_{k=1}^{p} \int_{\mathcal{R}} \frac{v_{k} e^{-\theta y}}{v_{i} \rho^{l}} Z_{\theta, k j}^{n-l}(A-y) Z_{i k}^{l}(d y) .
\end{aligned}
$$

By taking conditional expectation conditioning on $\mathcal{F}_{l}$ we get

$$
E\left[Z_{\theta, i j}^{n}(A) \mid \mathcal{F}_{l}\right]=\sum_{k=1}^{p} \sum_{y}\left[\frac{v_{k} e^{-\theta Z_{i k}^{l}(y)}}{v_{i} \rho^{l}}\right] \mu_{\theta, k j}^{(n-l) *}\left(A-Z_{i k}^{l}(y)\right) .
$$

By the central limit theorem for intensity measures in Rahimzadeh (2008) as $n-l \rightarrow \infty, \mu_{\theta, k j}^{(n-l) *}\left(A-Z_{i k}^{l}(y)\right)$ converges almost surely to a limit depending on $\theta$ and $k$. Replacing $\mu_{\theta, k j}^{(n-l) *}\left(A-Z_{i k}^{l}(y)\right)$ with its limit in (4) implies that $E\left[Z_{\theta, i j}^{n}(A) \mid \mathcal{F}_{l}\right]$ behaves asymptotically the same as the almost sure convergent martingale $\left\{w_{i}^{l}(\theta)\right\}$. This is the structure of the proof of Theorem 1. Before giving the proof of this theorem we give two definitions which are followed by a lemma. For any real number $\alpha \in(1,2]$, define $\sigma^{\alpha}(X)=E|X-E X|^{\alpha}$ and $\sigma^{\alpha}\left(X \mid \mathcal{F}_{l}\right)=E\left[\left|X-E\left(X \mid \mathcal{F}_{l}\right)\right|^{\alpha} \mid \mathcal{F}_{l}\right]$. The next lemma gives a bound for $\sigma^{\alpha}\left(. \mid \mathcal{F}_{l}\right)$.

Lemma 3 Let for some $\theta \in \Omega$, and $\alpha \in(1,2], \mathcal{A}(1)-\mathcal{A}(5)$ hold. Then there is a constant $C>0$, depending only on $\alpha$ and $\theta$, such that for all 
$i, j, l, n(1 \leqslant l \leqslant n)$, and any measurable set $A \subset \mathcal{R}$,

$$
\sigma^{\alpha}\left(Z_{\theta, i j}^{n}(A) \mid \mathcal{F}_{l}\right) \leqslant C \kappa^{l} W_{i}^{l}(\alpha \theta) .
$$

Proof. Let $1 \leqslant l \leqslant n$ and define

$$
X_{k, y}=Z_{\theta, k j}^{n-l}\left(A-Z_{i k}^{l}(y)\right) \quad \text { and } \quad c_{k, y}=\frac{v_{k} e^{-\theta Z_{i k}^{l}(y)}}{v_{i} \rho^{l}} .
$$

Then using (3) and (4) we can write

$$
\sigma^{\alpha}\left(Z_{\theta, i j}^{n}(A) \mid \mathcal{F}_{l}\right)=E\left[\left|\sum_{k, y} c_{k, y}\left[X_{k, y}-E\left[X_{k, y} \mid \mathcal{F}_{l}\right]\right]\right|^{\alpha} \mid \mathcal{F}_{l}\right] .
$$

Given $\mathcal{F}_{l}$, the terms $c_{k, y}$ are measurable and the terms inside the sum $\sum_{k, y}$, are independent with zero mean. Thus, by Lemma 2 in Biggins and Rahimzadeh (2005), for a constant $C_{1}$ depending only on $\alpha$ we have

$$
\begin{aligned}
\sigma^{\alpha}\left(Z_{\theta, i j}^{n}(A) \mid \mathcal{F}_{l}\right) & \leqslant C_{1} \sum_{k, y}\left|c_{k, y}\right|^{\alpha} \sigma^{\alpha}\left(X_{k, y} \mid \mathcal{F}_{l}\right) \\
& \leqslant 8 C_{1} \sum_{k, y}\left|c_{k, y}\right|^{\alpha} E\left[\left|X_{k, y}\right|^{\alpha} \mid \mathcal{F}_{l}\right]
\end{aligned}
$$

where in the second inequality we used the inequality $|a+b|^{\alpha} \leqslant 4\left(|a|^{\alpha}+\right.$ $\left.|b|^{\alpha}\right)$. Since $Z_{\theta, i j}^{n}(\cdot)$ is a nonnegatve measure and by Lemma 2 for $\alpha \in$ $(1,2], E\left|W_{i}^{n}(\theta)\right|^{\alpha}$ is bounded uniformly in $i$ and $n$, by $M_{1}$ say, then by conditioning on $\mathcal{F}_{l}$, we get

$$
\begin{aligned}
E\left[\left|X_{k, y}\right|^{\alpha} \mid \mathcal{F}_{l}\right] & =E\left[\left|Z_{\theta, k j}^{n-l}\left(A-Z_{i k}^{l}(y)\right)\right|^{\alpha} \mid \mathcal{F}_{l}\right] \\
& \leqslant E\left[\left|Z_{\theta, k j}^{n-l}(\mathcal{R})\right|^{\alpha} \mid \mathcal{F}_{l}\right] \\
& \leqslant E\left|W_{k}^{n-l}(\theta)\right|^{\alpha} \\
& \leqslant M_{1} .
\end{aligned}
$$

Let $M=\max _{i, k}\left\{v_{k}^{\alpha} v_{i}(\alpha \theta) / v_{i}^{\alpha} v_{k}(\alpha \theta)\right\}$ then by (5) and the previous inequality:

$$
\sigma^{\alpha}\left(Z_{\theta, i j}^{n}(A) \mid \mathcal{F}_{l}\right) \leqslant 8 C_{1} M_{1} \sum_{k, y}\left(\frac{v_{k}}{v_{i}}\right)^{\alpha} \frac{e^{-\alpha \theta Z_{i k}^{l}(y)}}{\rho^{\alpha l}}
$$




$$
\begin{aligned}
& =8 C_{1} M_{1}\left[\frac{\rho(\alpha \theta)}{\rho(\theta)^{\alpha}}\right]^{l} \sum_{k}\left[\left(\frac{v_{k}}{v_{i}}\right)^{\alpha} \frac{v_{i}(\alpha \theta)}{v_{k}(\alpha \theta)}\right]\left[\int_{\mathcal{R}} \frac{v_{k}(\alpha \theta) e^{-\alpha \theta y}}{v_{i}(\alpha \theta) \rho(\alpha \theta)^{l}} Z_{i k}^{l}(d y)\right] \\
& \leqslant 8 C_{1} M_{1} M \kappa^{l} W_{i}^{l}(\alpha \theta)
\end{aligned}
$$

where, $C=8 C_{1} M_{1} M$ is a constant depending only on $\alpha$ and $\theta$.

Now we give the proof of the main results.

Proof of Theorem 1 Let $A$ be a bounded measurable set in $\mathcal{R}$ and let $i, j$ be fixed. Let $\beta>3$ and for any $n$ define $l=\left[n^{1 / \beta}\right]$. We write

$$
\begin{aligned}
\mid \sqrt{2 \pi n} \sigma Z_{\theta, i j}^{n}(n a+A) & -u_{j} v_{j} W_{i}(\theta)|A| \mid \\
& \leq\left|\sqrt{2 \pi n} \sigma\left[Z_{\theta, i j}^{n}(n a+A)-E\left[Z_{\theta, i j}^{n}(n a+A) \mid \mathcal{F}_{l}\right]\right]\right| \\
& +\left|\sqrt{2 \pi n} \sigma E\left[Z_{\theta, i j}^{n}(n a+A) \mid \mathcal{F}_{l}\right]-u_{j} v_{j} W_{i}^{l}(\theta)\right| A|| \\
& +u_{j} v_{j}|A|\left|W_{i}^{l}(\theta)-W_{i}(\theta)\right| .
\end{aligned}
$$

Since $W_{i}(\theta)$ is the almost sure limit of $\left\{W_{i}^{l}(\theta)\right\}$, so the last term has zero limit, as $l \rightarrow \infty$. To prove the almost sure convergence of the first term to zero, let

$$
D_{n}=Z_{\theta, i j}^{n}(n a+A)-E\left[Z_{\theta, i j}^{n}(n a+A) \mid \mathcal{F}_{l}\right] .
$$

Since $\alpha>1$, from Jensen's inequality, conditioning on $\mathcal{F}_{l}$, we get $E\left[\mid D_{n} \| \mathcal{F}_{l}\right] \leqslant$ $\left[E\left[\left|D_{n}\right|^{\alpha} \mid \mathcal{F}_{l}\right]\right]^{\frac{1}{\alpha}}=\left[\sigma^{\alpha}\left(Z_{\theta, i j}^{n}(n a+A) \mid \mathcal{F}_{l}\right)\right]^{\frac{1}{\alpha}}$. By Lemma 3 we get

$$
E\left|D_{n}\right| \leqslant C^{(1 / \alpha)} \kappa^{(l / \alpha)} E\left[W_{i}^{l}(\alpha \theta)^{\frac{1}{\alpha}}\right] .
$$

Again by Jensen's inequality, $E\left[W_{i}^{l}(\alpha \theta)^{(1 / \alpha)}\right] \leqslant\left[E W_{i}^{l}(\alpha \theta)\right]^{(1 / \alpha)}=1$, so we have

$$
\sum_{n} \sqrt{n} E\left|D_{n}\right| \leqslant C^{(1 / \alpha)} \sum_{l} \sqrt{n} d_{l} \kappa^{(l / \alpha)}
$$

where, $d_{l}$ is the number of $n$ 's which are related to the fixed $l$ and is bounded by $d_{l} \leqslant(l+1)^{\beta}-l^{\beta}$. So we can continue 


$$
\begin{aligned}
\sum_{l} \sqrt{n} d_{l} \kappa^{(l / \alpha)} & \leqslant \sum_{l}(l+1)^{(\beta / 2)}\left((l+1)^{\beta}-l^{\beta}\right)\left(\kappa^{(1 / \alpha)}\right)^{l} \\
& =\sum_{l} P(l) \kappa_{1}^{l}<\infty
\end{aligned}
$$

where, $\kappa_{1}=\kappa^{(1 / \alpha)} \in(0,1)$ and $P(l)$ is a function of $l$ with $P(l)=O\left(l^{(3 \beta / 2)}\right)$, as $l \rightarrow \infty$. Thus the series on the left hand side of (7) converges, and then by the well known results we get the convergence of $\sqrt{n} E\left|D_{n}\right| \rightarrow 0$, as $l, n \rightarrow \infty$. This implies the almost sure convergence of $\left|\sqrt{n} D_{n}\right|$ or the convergence of the first part on the right hand of (6) to 0 , as $l, n \rightarrow \infty$.

Now we turn to prove the zero limit of the second part in 6 . Let $f_{\sigma}$ be the density function of $\mathrm{N}\left(0, \sigma^{2}\right)$, then we can write

$$
\begin{aligned}
\left|\sqrt{2 \pi n} \sigma E\left[Z_{\theta, i j}^{n}(n a+A) \mid \mathcal{F}_{l}\right]-u_{j} v_{j} W_{i}^{l}(\theta)\right| A|| \\
\leqslant \frac{\sqrt{2 \pi n}}{\sqrt{n-l}} \sigma \mid \sqrt{n-l} E\left[Z_{\theta, i j}^{n}(A+n a) \mid \mathcal{F}_{l}\right] \\
\quad-u_{j} v_{j} \sum_{k, y} \frac{v_{k} e^{-\theta Z_{i k}^{l}(y)}}{v_{i} \rho^{l}} \int_{A+l a-Z_{i k}^{l}(y)} f_{\sigma}\left(\frac{x}{\sqrt{n-l}}\right) d x \mid \\
+\frac{\sqrt{2 \pi n}}{\sqrt{n-l}} \sigma u_{j} v_{j} \sum_{k, y} \frac{v_{k} e^{-\theta Z_{i k}^{l}(y)}}{v_{i} \rho^{l}} \\
\quad \times\left|\int_{A+l a-Z_{i k}^{l}(y)}\left[f_{\sigma}\left(\frac{x}{\sqrt{n-l}}\right)-\frac{1}{\sqrt{2 \pi} \sigma}\right] d x\right| \\
+\frac{\sqrt{n}-\sqrt{n-l}}{\sqrt{n-l}} u_{j} v_{j} W_{i}^{l}(\theta)|A| \\
=E_{n}+F_{n}+G_{n}, \quad \text { (say) }
\end{aligned}
$$

Since $l=o(n)$, by Proposition 1 below the second part on the right hand side of (8) tends to zero, almost surely as $l, n \rightarrow \infty$. In the last part, $G_{n}$, the fraction $(\sqrt{n}-\sqrt{n-l}) / \sqrt{n-l}$, has also zero limit by $l=o(n)$ and $\left\{W_{i}^{l}(\theta)\right\}$ is an almost sure convergent martingale, hence the last part converges to zero almost surely. To prove the zero limit of first part in (8), $E_{n}$, from Lemma 1 we have

$$
\lim _{n \rightarrow \infty}\left|\sqrt{n} g_{i j}^{n}(x)-f_{\sigma}\left(\frac{x}{\sqrt{n}}\right)\right|=0 \quad \text { as } \quad n \rightarrow \infty
$$


uniformly in $x \in \mathcal{R}$, where $g_{i j}^{n}$ is the probability density function of $\bar{\mu}_{\theta, i j}^{n *} / p_{i j}^{n}$. Then by integrating over bounded set $B$ with $|B|<2 b$ we get, as $n \rightarrow \infty$,

$$
\sup _{|B|<2 b}\left|\frac{\sqrt{n}}{p_{i j}^{n}} \bar{\mu}_{\theta, i j}^{n *}(B)-\int_{B} f_{\sigma}\left(\frac{x}{\sqrt{n}}\right) d x\right| \rightarrow 0 .
$$

Since $p_{i j}^{n} \rightarrow u_{j} v_{j}$ as $n \rightarrow \infty$, we get

$$
\delta_{n}=: \max _{i, j} \sup _{|B|<2 b}\left|\sqrt{n} \bar{\mu}_{\theta, i j}^{n *}(B)-u_{j} v_{j} \int_{B} f_{\sigma}\left(\frac{x}{\sqrt{n}}\right) d x\right| \rightarrow 0 .
$$

Since $l=o(n)$, by using the decomposition of $E\left[Z_{i k}^{n}(A) \mid \mathcal{F}_{l}\right]$ from the equation (4) and applying the previous inequality we can write

$$
\begin{aligned}
E_{n} & \leqslant \sqrt{2 \pi} \sigma \frac{\sqrt{n}}{\sqrt{n-l}}\left[\sum_{k, y} \frac{v_{k} e^{-\theta Z_{i k}^{l}(y)}}{v_{i} \rho^{l}} \mid \sqrt{n-l} \bar{\mu}_{\theta, k j}^{(n-l) *}\left(A+l a-Z_{i k}^{l}(y)\right)\right. \\
& \left.-u_{j} v_{j} \int_{A+l a-Z_{i k}^{l}(y)} f_{\sigma}\left(\frac{x}{\sqrt{n-l}}\right) d x \mid\right] \\
& \leqslant \sqrt{2 \pi} \sigma \delta_{n-l} \frac{\sqrt{n}}{\sqrt{n-l}}\left[W_{i}^{l}(\theta)\right] .
\end{aligned}
$$

Since $\left\{W_{i}^{n}(\theta)\right\}$ is an almost sure convergent martingale and $(\sqrt{n} / \sqrt{n-l}) \rightarrow$ 1 by $l=o(n)$, these imply that the right hand side tends to zero, as $n \rightarrow \infty$, and this completes the proof.

Proposition 1 Suppose the hypotheses of Theorem 1 hold. Let $\beta>3, l=$ $\left[n^{1 / \beta}\right]$ and $i, j$ be fixed. Then for any bounded measurable set $A \subset[-b, b]=I$, as $n \rightarrow \infty$,

$$
H_{n}(A)=\sum_{k, y} \frac{v_{k} e^{-\theta Z_{i k}^{l}(y)}}{v_{i} \rho^{l}} \int_{A+l a-Z_{i k}^{l}(y)}\left|f_{\sigma}\left(\frac{x}{\sqrt{n-l}}\right)-\frac{1}{\sigma \sqrt{2 \pi}}\right| d x
$$

converges to zero almost surely, where $f_{\sigma}$ is the density of the normal distribution $\mathrm{N}\left(0, \sigma^{2}\right)$ and $\sigma^{2}=(\log \rho(\theta))^{\prime \prime}>0$.

Proof. Let $f_{\sigma}$ be the density function of normal distribution $\mathrm{N}\left(0, \sigma^{2}\right)$. Then there is a constant $C>0$, such that $\left|f_{\sigma}(x)-1 /(\sigma \sqrt{2 \pi})\right| \leqslant C x^{2}$, for all $x \in \mathcal{R}$. Let also $A$ be a bounded measurable set with $A \subset[-b, b]$ then $A+l a-Z_{i k}^{l}(y) \subset I+l a-Z_{i k}^{l}(y)$ and this implies that 


$$
\begin{aligned}
E H_{n}(A) & \leqslant C E \sum_{k, y} \frac{v_{k} e^{-\theta Z_{i k}^{l}(y)}}{v_{i} \rho^{l}} \int_{A+l a-Z_{i k}^{l}(y)}\left(\frac{x}{\sqrt{n-l}}\right)^{2} d x \\
& \leqslant \frac{C_{2}}{3(n-l)} \sum_{k} \frac{v_{k}}{v_{i} \rho^{l}} E \int_{\mathcal{R}} e^{-\theta y}\left[2 b^{3}+6 b(l a-y)^{2}\right] Z_{i k}^{l}(d y) \\
& \leqslant C^{\prime} \sum_{k} \frac{v_{k}}{v_{i} \rho^{l}} \int_{\mathcal{R}} e^{-\theta y}\left[2 b^{3}+6 b(l a-y)^{2}\right] \mu_{i k}^{l *}(d y) \\
& \leqslant \frac{C^{\prime}}{v_{i} \rho^{l}} \sum_{k} v_{k} \int_{\mathcal{R}} e^{-\theta y}\left[\left(2 b^{3}+6 b l^{2} a^{2}\right)-12 a b l y+6 b y^{2}\right] \mu_{i k}^{l *}(d y)
\end{aligned}
$$

where $C^{\prime}=C_{2} / 3(n-l)$. The functions $v_{i}, u_{j}, \rho$, and $m_{i j}(\theta)$ are analytic and $\left\{\rho(\theta)^{-n} m_{i j}^{n}(\theta)\right\}$ is a convergent sequence, hence is bounded by some $M_{1}>0$ say. The first two derivatives of the equation $M^{n}(\theta) v(\theta)=\rho^{n}(\theta) v(\theta)$ imply that, for some constants $c_{1}, \ldots, c_{5}$, we have $\sum_{j}\left(m_{i j}^{n}(\theta)\right)^{\prime} v_{j} \leqslant\left(c_{1} n+c_{2}\right) \rho^{n}$ and $\sum_{j=1}^{p}\left(m_{i j}^{n}(\theta)\right)^{\prime \prime} v_{j} \leqslant\left(c_{3} n^{2}+c_{4} n+c_{5}\right) \rho^{n}$. So for some constants $M_{2}$ and $M_{3}, \quad \sum_{j=1}^{p}\left(m_{i j}^{n}(\theta)\right)^{\prime} v_{j} \leqslant M_{2} n \rho^{n}$ and $\sum_{j=1}^{p}\left(m_{i j}^{n}(\theta)\right)^{\prime \prime} v_{j} \leqslant M_{3} n^{2} \rho^{n}$. Now we can continue (9) to write

$$
\begin{aligned}
\ldots & \leqslant \frac{C^{\prime}}{v_{i} \rho^{l}} \sum_{k} v_{k}\left[\left(2 b^{3}+6 b l^{2} a^{2}\right) m_{i k}^{l}(\theta)+12|a| b l\left(m_{i k}^{l}(\theta)\right)^{\prime}+6 b\left(M_{i k}^{l}(\theta)\right)^{\prime \prime}\right] \\
& \leqslant \frac{C^{\prime}}{v_{i} \rho^{l}}\left[\left(2 b^{3}+6 b l^{2} a^{2}\right) M_{1} \rho^{l}+12|a| b l^{2} M_{2} \rho^{l}+6 b l^{2} M_{3} \rho^{l}\right] \\
& \leqslant \frac{C_{2} M l^{2}}{3(n-l) v_{i}}
\end{aligned}
$$

for some constant $M$. Since $l=\left[n^{1 / \beta}\right]$ with $\beta>3$ then the right hand side of (9) converges to zero and hence $E H_{n} \rightarrow 0$. This implies that $H_{n} \rightarrow 0$ almost surely as $l, n \rightarrow \infty$ and completes the proof.

We apply the Riemman integral estimate to Theorem 1 to prove our second main result.

Proof of Theorem 2 Let $A \subset[-b, b]=I$ be a measurable set. For any fixed $i, j$, and $n$, from the definition of $Z_{i j}^{n}(\cdot, \theta)$ we can write 


$$
\begin{aligned}
Z_{i j}^{n}(n a+A) & =\int_{n a+A}\left[\frac{v_{i}}{v_{j}} e^{\theta x} \rho^{n}\right]\left[\frac{v_{j}}{v_{i}} \frac{e^{-\theta x}}{\rho^{n}} Z_{i j}^{n}(d x)\right] \\
& =\left[\frac{v_{i}}{v_{j}} \rho^{n}\right] \int_{n a+A} e^{\theta x} Z_{\theta, i j}^{n} d x
\end{aligned}
$$

By changing the variable $x$ to $x+n a$ and recalling that $a=-\rho^{\prime}(\theta) / \rho(\theta)$, we get

$$
Z_{i j}^{n}(n a+A)=\frac{v_{i}}{v_{j}} e^{n \Lambda^{*}(a)} \int_{A} e^{\theta x} \bar{Z}_{\theta, i j}^{n} d x .
$$

Define $g(x)=1_{A}(x) e^{\theta x}$ for all $x \in I$. Let $\left\{A_{r}: r\right\}$ be a finite partition of $I$, then for each $r$, define $M_{r}=\sup \left\{g(x): x \in A_{r}\right\}$ and $m_{r}=\inf \{g(x): x \in$ $\left.A_{r}\right\}$. For each $x \in I$, define $\bar{g}(x)=\sum_{r} M_{r} 1_{A_{r}}(x)$ and $\underline{g}(x)=\sum_{r} m_{r} 1_{A_{r}}(x)$. Then for any $x \in A, \underline{g}(x) \leqslant g(x) \leqslant \bar{g}(x)$. From (10) we have

$$
Z_{i j}^{n}(n a+A) \leqslant \frac{v_{i}}{v_{j}} e^{n \Lambda^{*}(a)} \sum_{r} M_{r} \bar{Z}_{\theta, i j}^{n}\left(A_{r}\right) .
$$

Let $M=\sum_{r} M_{r}$ and $\epsilon>0$ be fixed. From Theorem 1, for any $r$, there is an $N_{1}$ such that, for all $n \geqslant N_{1}$, we have

$$
\left|\sqrt{2 \pi n} \sigma \bar{Z}_{\theta, i j}^{n}\left(A_{r}\right)-u_{j} v_{j} W_{i}(\theta)\right| A_{r}|| \leqslant \epsilon / M
$$

We choose $N_{1}$ big enough such that (12) holds for all $r$. Then for any $n \geqslant N_{1}$, by applying (12) in (11), we can continue

$$
\begin{aligned}
\sqrt{2 \pi n} \sigma Z_{i j}^{n}(n a+A) & \leqslant\left[\frac{v_{i}}{v_{j}} e^{n \Lambda^{*}(a)}\right] \sum_{r} M_{r}\left[u_{j} v_{j} W_{i}(\theta)\left|A_{r}\right|+\epsilon / M\right] \\
& =\left[\frac{v_{i}}{v_{j}} e^{n \Lambda^{*}(a)}\right]\left[L \int_{A} \bar{g}(x) d x+\epsilon\right]
\end{aligned}
$$

where, $L=u_{j} v_{j} W_{i}(\theta)$. Thus, for any $\epsilon>0$, we have

$$
J_{n}=: \sqrt{2 \pi n} \sigma \frac{v_{j}}{v_{i}} e^{-n \Lambda^{*}(a)} Z_{i j}^{n}(n a+A) \leqslant L \int_{A} \bar{g}(x) d x+\epsilon .
$$

By taking limsup of (13), as $n \rightarrow \infty$, since $\epsilon$ is arbitrary, we get

$$
\limsup _{n \rightarrow \infty} J_{n} \leqslant L \int_{A} \bar{g}(x) d x .
$$


The equation (14) holds for all partitions of $I$, thus by taking infimum on all partitions of $I$ and taking into account that, $J_{n}$ is independent of these partitions, we get

$$
\limsup _{n \rightarrow \infty} J_{n} \leqslant L \int_{A} g(x) d x
$$

With a similar argument we can prove that

$$
\lim \inf _{n \rightarrow \infty} J_{n} \geqslant L \int_{A} g(x) d x .
$$

Then (15) and (16) completes the proof.

\section{Acknowledgement}

The author would like to thank Professor J. D. Biggins for many valuable discussions.

\section{References}

Biggins, J. D. (1992). Uniform convergence of martingales in the branching random walk. Ann. Probab. 20, 137-151.

Biggins, J. D. and Rahimzadeh Sani, A. (2005). Convergence results on multitype, multivariate branching random walks. Adv. Appl. Probab. 37, 681-705.

Bramson, M., Ney, P. and Tao, J. (1992). The population composition of a multitype branching random walk. Ann. Appl. Probab. 2, 575-596.

Mode, C.J. (1971). Multitype Branching Processes. American Elsevier, New York.

Rahimzadeh Sani, A. (2008). Local And Centra Limit Theorems For Intesity Measures In Multitype Branching Random Walk. J. Sci. Tarbiat Moallem University. 7, No. 3, 4, 233240.

\section{A. Rahimzadeh Sani}

Department of Mathematics, Tarbiat Moallem University, Tehran, Iran.

email: rahimsan@saba.tmu.ac.ir 